\title{
Experimental Demonstration of Dual O+C-band WDM Transmission over 50-km SSMF with Direct Detection
}

\author{
Yang Hong, Member, IEEE, Kyle R. H. Bottrill, Natsupa Taengnoi, Naresh K. Thipparapu, Yu Wang, \\ Andrey A. Umnikov, Jayanta K. Sahu, David J. Richardson, Fellow, IEEE and \\ Periklis Petropoulos, Fellow, OSA
}

\begin{abstract}
In this paper, we experimentally demonstrate dual $\mathrm{O}+\mathrm{C}$-band direct-detection wavelength division multiplexed (WDM) transmission over $50 \mathrm{~km}$ of standard single-mode fibre, wherein $4 \times 40-\mathrm{Gb} / \mathrm{s}$ Nyquist on-off keying (OOK) modulation is used in the O-band whilst $4 \times 40-\mathrm{Gb} / \mathrm{s}$ single-sideband (SSB) subcarrier modulation (SCM) with Kramers-Kronig (KK) detection is used in the C-band. A bismuth-doped fibre amplifier (BDFA) is used as the pre-amplifier in the O-band to extend the reach of the direct-detection system. It is demonstrated that the $O$-band exhibits superior receiver sensitivity relative to the $\mathrm{C}$-band and its transmission performance is limited by the chromatic dispersion (CD), especially when longer reaches and wavelengths in the longer edge of the band are explored. In contrast, due to the adoption of the SSB format, the C-band shows enhanced tolerance to $C D$ effects. In this case, some receiver sensitivity is sacrificed to accommodate the adoption of the KK detection which is used to eliminate the signal-to-signal beating interference of the SSB-SCM format. The inferior receiver sensitivity in the C-band is caused by (1) the higher CSPR requirement of the $\mathrm{KK}$ detection and (2) the restriction of small-signal modulation for the dual-drive Mach-Zehnder modulator to generate optical SSB signals. The results indicate the feasibility to further extend the reach of $\mathrm{O}$-band transmission systems, which enables the possibility to realise dual $\mathrm{O}+\mathrm{C}$-band WDM transmission in intermediate- to long-haul optical networks.
\end{abstract}

Index Terms-Optical communications, Point-to-point transmission, Ultra-wideband WDM systems.

\section{INTRODUCTION}

$\mathrm{O}$ WING to the increased popularity of multimedia streaming and the emergence of numerous new applications, such as virtual reality and augmented reality, an

Manuscript was received May 31, 2019. This work was supported by the UK's EPSRC under the Airguide Photonics Programme grant (EP/P030181/1) and the PHOS project (EP/S002871/1) (Corresponding author: Yang Hong).

The authors are with the Optoelectronics Research Centre, University of Southampton, Southampton SO17 1BJ, UK. (e-mails: y.hong@soton.ac.uk; k.bottrill@soton.ac.uk; ntla15@soton.ac.uk; nkt1d17@soton.ac.uk; yw9n17@soton.ac.uk; a.a.umnikov@soton.ac.uk; jks@orc.soton.ac.uk; djr@orc.soton.ac.uk; pp@orc.soton.ac.uk)

Color versions of one or more of the figures in this paper are available online at http://ieeexplore.ieee.org.

Digital Object Identifier: XXXXXXX ever-increasing demand for high data rate transmission in data centre interconnects and point-to-point metro networks has been witnessed [1-2]. Considering that the reach for such systems tends to be in the range $40 \mathrm{~km}$ to $80 \mathrm{~km}$, low-cost direct-detection (DD) solutions are more favourable over coherent detection. This is mainly because the current implementation costs of coherent systems are still very much higher than that of DD solutions, even though coherent solutions offer superior receiver sensitivity, extended reach and enhanced spectral efficiency (SE) [3]. To take advantage of the inherent simplicity of DD implementations, while making DD solutions more competitive relative to the coherent detection, substantial efforts have been made to narrow down the performance gap.

One promising solution for extending the reach and improving the SE of DD systems is to adopt single-sideband (SSB) formats, which have demonstrated their capabilities in intermediate- to long-haul optical transmission systems [4-6]. The SE of optical systems based on SSB formats can be doubled as compared to that of systems using double-sideband (DSB) formats in a straightforward manner. Furthermore, SSB formats do not suffer from the RF power fading issue induced by chromatic dispersion (CD), which is the limiting issue for DSB format based DD systems when longer reach or higher capacity is required. However, despite the aforementioned advantages, SSB modulation suffers from inherent signal-to-signal beating interference (SSBI), which results from the unavoidable nonlinear distortion when directly detecting optical SSB signals [7]. In the literature, various digital signal processing (DSP) schemes have been proposed to avoid or mitigate SSBI. A guard band based scheme was proposed in [4], wherein a guard band of a bandwidth equal to that of the signal is inserted between the optical carrier and the optical SSB signal, such that SSBI can be avoided at the expense of halving the SE. Unlike the guard band based scheme, and without sacrificing the SE, in [5] the SSBI was first approximately reconstructed and then subtracted from the detected signal to provide for SSBI cancellation, however the results showed that a relatively large carrier-to-signal power ratio (CSPR) was required for the SSBI reconstruction. Alternatively, linearization filter based schemes were also proposed to estimate and then cancel the SSBI contributions [7-8]. While 
better transmission performance can be attained by both two-stage and iterative linearization filter based schemes [8], the corresponding implementation complexity is also much higher. Recently, the Kramers-Kronig (KK) receiver has attracted much interest, since it is capable of completely eliminating SSBI without requiring an excessively large CSPR [9]. The KK detection was adopted in a C-band wavelength division multiplexing (WDM) DD system in [8], wherein better transmission performance was demonstrated compared to other SSBI cancellation schemes. Further improvements in KK detection regarding the relaxation of the up-sampling and CSPR requirements were reported in [10-12], showing a promising way to extend reach and to augment the achievable capacity of DD systems.

In addition to advanced DSP techniques, the research community is also keenly interested in exploring other bands within the transmission window of standard single-mode fibre (SSMF) for potential WDM transmission [13]. An ultra-wideband WDM system across the $\mathrm{S}+\mathrm{C}+\mathrm{L}-$ band was reported in [14], wherein 40-km SSMF transmission was achieved by using coherent detection. In [15], a 5-band (O, E, S, $\mathrm{C}$, and L) WDM coherent transmission over $60-\mathrm{km}$ of SSMF was demonstrated and wavelength adaptive modulation format allocation was used to accommodate the wavelength-dependent loss of the different bands. Arguably, the O-band is a more favourable choice since it has been specified as the transmission window for most short-haul optical systems $(<40$ $\mathrm{km})$ [16]. However, further extension of the reach of O-band systems remains a challenge, which makes the O-band less competitive than the C-band. The difficulty in O-band's reach extension is mainly attributed to the relatively high noise figure, polarisation sensitivity, and signal distortion at high speeds of the conventional semiconductor optical amplifier (SOA), which is the dominant commercially available O-band amplification technology at present [17]. One recent significant development in the search for a better O-band amplifier is the emergence of the bismuth-doped fibre amplifier (BDFA) [18-19], which is considered a promising technology for achieving long-haul O-band transmission. A recent demonstration of coarse WDM (CWDM) and dense WDM (DWDM) transmission over up to $140-\mathrm{km}$ SSMF showed negligible transmission penalties from the use of an inline BDFA $[17,20]$, highlighting the potential of the technology.
In this paper, we report $320-\mathrm{Gb} / \mathrm{s}$ dual $\mathrm{O}+\mathrm{C}-$ band direct-detection transmission over 50-km of SSMF using eight WDM optical channels. In the C-band, $4 \times 40-\mathrm{Gb} / \mathrm{s} 4$-ary quadrature amplitude modulation (4QAM) based SSB-subcarrier modulation (SSB-SCM) with KK detection is employed to combat the $\mathrm{CD}$ and SSBI issues. $4 \times 40-\mathrm{Gb} / \mathrm{s}$ Nyquist on-off keying (OOK) transmission is adopted in the O-band, considering its much lower $\mathrm{CD}$ which facilitates the usage of simpler DSB formats (e.g., Nyquist-OOK) rather than the relatively complicated SSB format. A BDFA is used as a pre-amplifier at the receiver in the O-band. We show that the O-band exhibits a better receiver sensitivity than the C-band, whereas the $\mathrm{CD}$ is a limiting factor for O-band transmission with longer wavelengths and longer reach. Thanks to the SSB modulation, superior CD tolerance is achieved in the C-band, however the receiver sensitivity is somewhat sacrificed to accommodate the SSBI cancellation for the optical SSB signals using KK detection.

\section{EXPERIMENTAL SETUP}

Fig. 1 shows the experimental setup of the dual $\mathrm{O}+\mathrm{C}$-band WDM DD system. In the C-band, four distributed feedback (DFB) lasers operating at $1549.63 \mathrm{~nm}, 1549.93 \mathrm{~nm}, 1550.23$ $\mathrm{nm}$ and $1550.53 \mathrm{~nm}$ were combined together via $3-\mathrm{dB}$ optical couplers. The combined signal was then fed into a dual-drive Mach-Zehnder modulator (DD-MZM) for optical SSB signal modulation. Note that the reason for using the DD-MZM instead of the IQ modulator (IQM) in this work is that the DD-MZM based approach has a simpler configuration and easier implementation and offers costs as low as $1 / 3$ that of the IQM-based scheme [21], which makes it more attractive for cost-sensitive DD systems. The main idea of such optical SSB signal generation is to bias the DD-MZM at its quadrature point and to modulate it with a relatively small optical modulation index (OMI). The corresponding principles were detailed in [21]. At the output of the DD-MZM, a waveshaper was used to de-multiplex the odd and even channels for de-correlation via transmission along different lengths of fibre. Subsequently, the signals were re-combined and fed into a booster erbium-doped fibre amplifier (EDFA). The output power of the EDFA was fixed at $2 \mathrm{dBm}$. Fig. 2 shows the corresponding bit error rate (BER) and signal-to-noise ratio (SNR) performances of the 1550.53-nm channel under different OMI when the ROP was

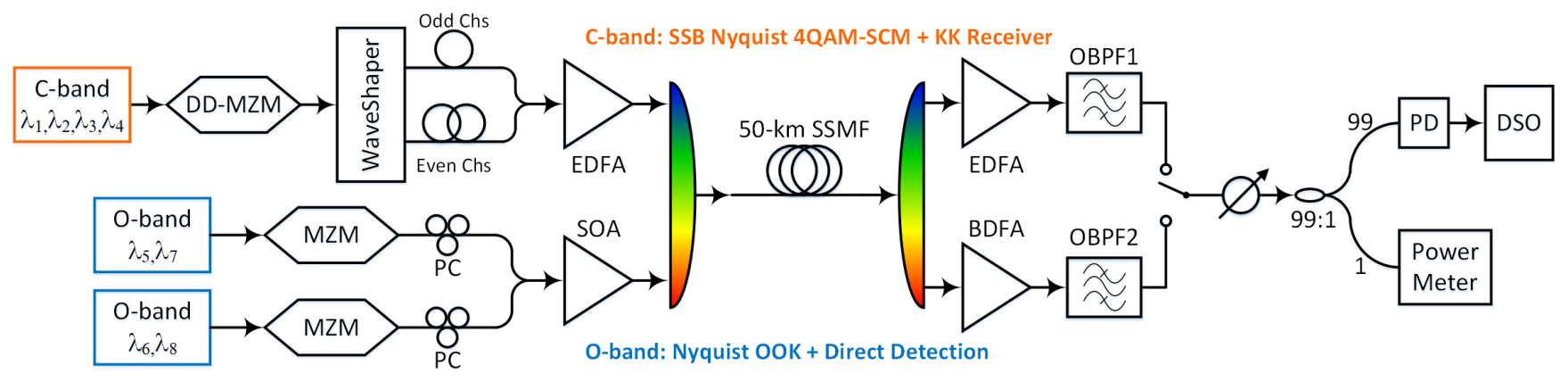

Fig. 1. Experimental setup of the dual O+C-band WDM transmission over 50-km SSMF. 
$-1.5 \mathrm{dBm}$. It is clear that at an OMI of around 0.194, the optimal BER and SNR performances can be achieved. With the optimized OMI, the optical spectra of the de-correlated optical SSB signals (before the booster EDFA), which were captured with a resolution of $0.01 \mathrm{~nm}$, are shown in Fig. 3. The CSPR of the optical SSB signals was $\sim 11 \mathrm{~dB}$, which corresponded to the optimized OMI, i.e., around 0.194, for the DD-MZM used.

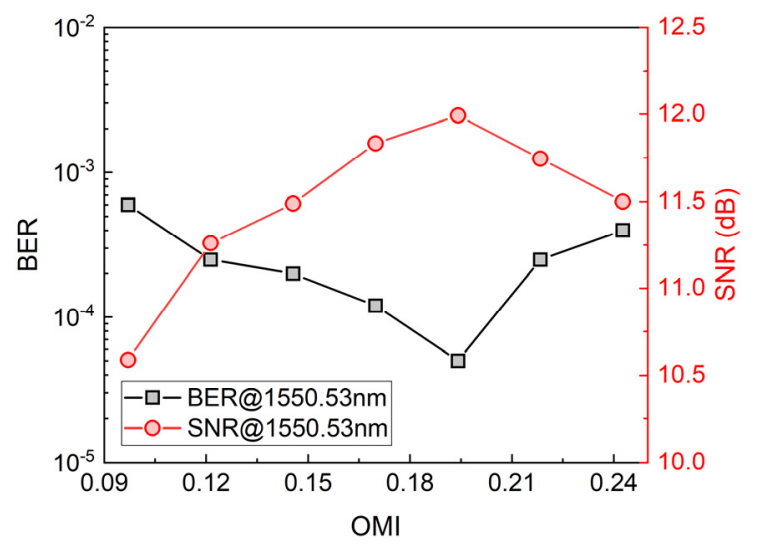

Fig. 2. BER and SNR versus OMI of the $1550.53-\mathrm{nm}$ channel at a ROP of -1.5 $\mathrm{dBm}$.

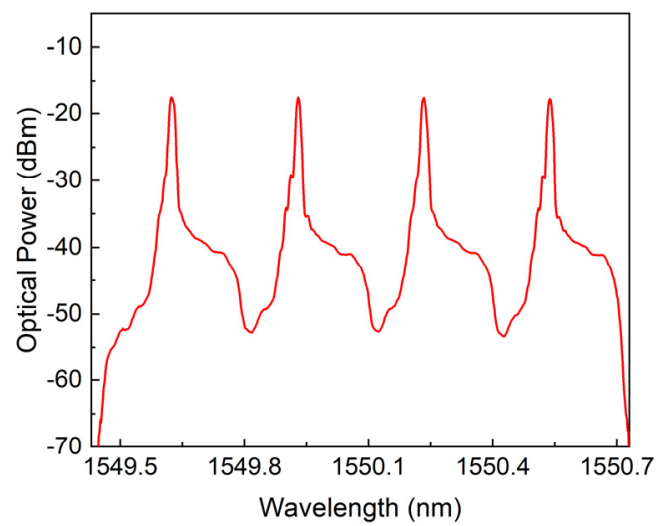

Fig. 3. Optical spectra of the de-correlated SSB-4QAM-SCM signals in the C-band.

In the O-band, four DFB lasers were used to generate optical carriers at $1330.92 \mathrm{~nm}, 1341.64 \mathrm{~nm}, 1351.32 \mathrm{~nm}$ and 1356.12 $\mathrm{nm}$. Then, two odd and two even optical carriers were injected into two separate MZMs for optical modulation. The modulated optical signals were combined together and then fed into an O-band SOA to boost the launch power. Note that the states of polarisation of the two MZM outputs was adjusted using polarisation controllers (PCs) to account for the polarisation sensitivity of the SOA. To compensate for the higher loss in the O-band after the 50-km length of SSMF, the output power of the SOA was fixed at $8 \mathrm{dBm}(6-\mathrm{dB}$ higher than the C-band's launch power). Finally, the amplified optical signals in the two bands were multiplexed via a WDM coupler and launched into a 50-km length of fibre for dual-band WDM transmission.

At the receiver, the optical signals of the two bands were first de-multiplexed by another WDM coupler. An EDFA was used to pre-amplify the C-band channels. Then, a tunable filter with a bandwidth of $0.3 \mathrm{~nm}$ was used to select the desired channel for performance evaluation. In the O-band, a BDFA was used as the pre-amplifier.

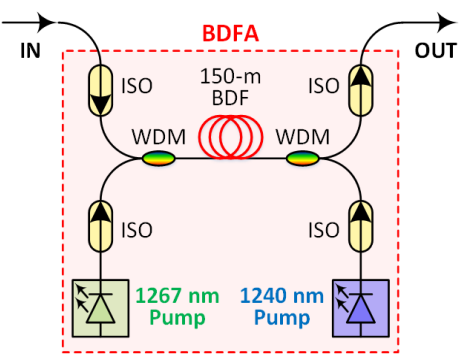

Fig. 4. Diagram of the implementation of the BDFA.

The schematic diagram of the BDFA is illustrated in Fig. 4, and it is composed of a 150-m length of phosphosilicate BDF, a 1267-nm pump laser, a 1240-nm pump laser, four optical isolators (ISO), and two WDM multiplexers. The two pump lasers were used to bi-directionally pump the BDF and the total combined pump power was around $720 \mathrm{~mW}$. With this configuration, $>20-\mathrm{dB}$ gain can be obtained for wavelengths ranging from $\sim 1320 \mathrm{~nm}$ to $\sim 1370 \mathrm{~nm}$, as shown in Fig. 5 . The saturated output power of the BDFA was around $16 \mathrm{dBm}$ whilst its noise figure was approximately $4.5 \mathrm{~dB}$. A more detailed characterization of the BDFA can be found in [19]. For comparison, the gain profile of the SOA is also shown in Fig. 5. It is seen that for the wavelengths investigated, the gain profiles of both the BDFA and the SOA are relatively uniform. Furthermore, it is worth noting that the SOA's nonlinearity has the potential to limit the performance of the O-band transmission. In contrast, while offering a higher gain, the BDFA also exhibits reduced nonlinearity, which facilitates enhanced transmission performance both in the B2B and the $60-\mathrm{km}$ SSMF link, as has been reported in [22].

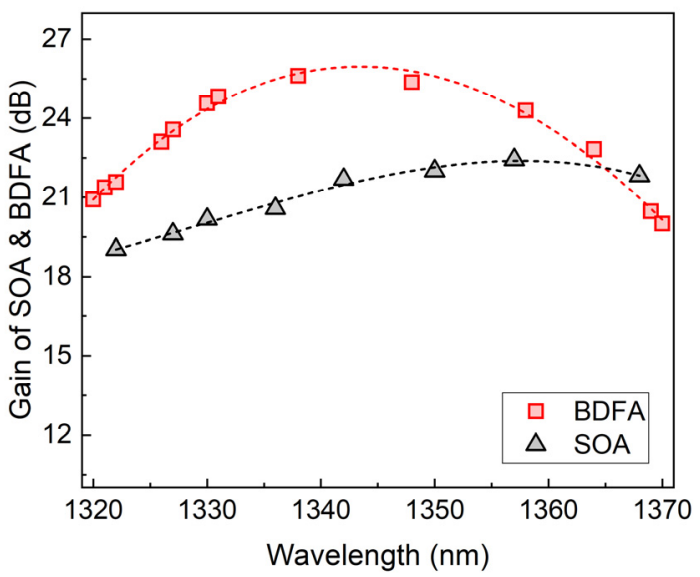

Fig. 5. The gain profiles of the BDFA and the SOA with an optical input power of $-20 \mathrm{dBm}$.

At the BDFA output, a tunable filter with a bandwidth of 1.2 $\mathrm{nm}$ was adopted for optical filtering and channel selection. For both bands, an optical attenuator was used to vary the received optical power (ROP) and a 99:1 optical coupler was used to monitor the ROP at the DC-coupled photodiode (PD, Finisar XPDV2320R). Note that according to the specification of the 
$\mathrm{PD}$, the responsivities of the $\mathrm{PD}$ at $1310 \mathrm{~nm}$ and $1550 \mathrm{~nm}$ are $0.45 \mathrm{~A} / \mathrm{W}$ and $0.65 \mathrm{~A} / \mathrm{W}$, respectively. The detected signal at the PD was then captured by a digital storage oscilloscope (DSO) for further offline DSP.

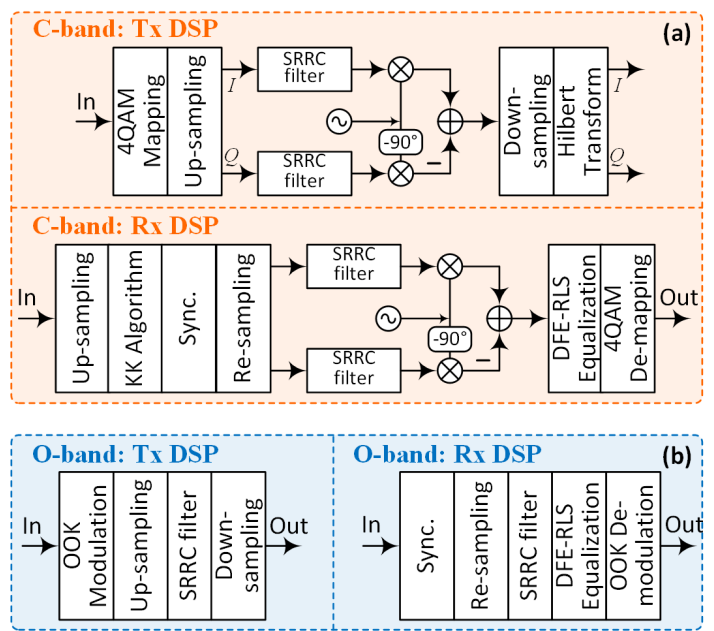

Fig. 6. The block diagrams of the offline DSP: (a) C-band and (b) O-band.

The block diagrams of the offline DSP used for the two bands are illustrated in Fig. 6. At the transmitter, in the C-band, the input binary bits were first mapped to 4QAM symbols, followed by an up-sampling operation. The in-phase $(I)$ and quadrature $(Q)$ components of the up-sampled signal were then filtered by a square-root raised cosine (SRRC) filter with a roll-off factor of 0.1 for Nyquist shaping. Subsequently, to realise half-cycle SCM, the centre frequencies of the shaped $I$ and $Q$ components were shifted to 0.52 of the symbol rate by applying a Sine carrier and Cosine carrier, respectively. Finally, the two components were added together to generate the DSB Nyquist SCM signal. The resulting signal was then down-sampled and converted to a SSB Nyquist SCM signal by using a digital Hilbert transform operation. The $I$ and $Q$ components of the generated SSB signal were then sent to two separate channels of a four-channel arbitrary waveform generator (AWG) with a fixed sampling rate of $90 \mathrm{GSa} / \mathrm{s}$ and were used to drive the two arms of the DD-MZM. As shown in Fig. 6(b), an identical SRRC filter was used to shape the up-sampled OOK signal of the O-band channels. The shaped signal was then down-sampled to generate the $40-\mathrm{Gb} / \mathrm{s}$ Nyquist OOK signal with a 90-GSa/s AWG. Note that to avoid correlation, two individual Nyquist-OOK signals were generated for the O-band and used to drive the two MZMs independently. We note that the reason for adopting OOK as the modulation format in the O-band, (instead of a more efficient modulation format, such as 4-ary pulse amplitude modulation (PAM4)), is that OOK signals offer enhanced tolerance to the nonlinearity of the SOA, which facilitates a better BER performance under relatively low SNR conditions.

At the receiver, the signals of the two bands were captured with an $80-\mathrm{GSa} / \mathrm{s}$ digital storage oscilloscope (DSO). For the C-band, the recorded signal was first six times up-sampled to accommodate the bandwidth broadening induced by the square-law detection and the logarithmic operation. Then, the complex field of the signal was reconstructed by applying the $\mathrm{KK}$ algorithm, which is summarised as

$$
\begin{aligned}
& \phi_{E}(t)=H\left[\ln \left(\sqrt{I_{P D}(t)}\right)\right] \\
& E(t)=\sqrt{I_{P D}(t)} \exp \left(j \phi_{E}(t)\right)
\end{aligned}
$$

where $\phi_{E}(t)$ and $I_{P D}(t)$ denote the phase of the complex field and the intensity of the detected current, respectively; $H[\cdot]$ is a Hilbert transform operator; and $E(t)$ is the recovered full-field complex signal. Following the KK algorithm, synchronization and matched filtering were then applied to the SSBI-compensated signal. After that, a T/2-spaced (17, 7)-tap decision-feedback equalizer (DFE) using the recursive least squares (RLS) algorithm was adopted. The forgetting factor of the RLS algorithm was 0.99 . Finally, the equalized signal was de-mapped and the bit error rate (BER) of the transmission was attained via error counting. The offline DSP at the receiver of the O-band signals is much simpler, as shown in Fig. 6(b). Up-sampling and $\mathrm{KK}$ detection were excluded from the processing of the DSB Nyquist-OOK signal. After synchronization, the signal was re-sampled and matched filtered, followed by an RLS-based DFE, whose setting was identical to that used in the C-band. We note that although the RLS-based DFE was implemented to compensate the channel response of the transmission line, it cannot resolve the issue of chromatic dispersion (CD) induced transmission performance degradation. As will be shown in the following section, the transmission performance of the longer wavelength O-band channels will degrade due to the higher fiber CD.

The main targets of these experiments presented in this work are to provide useful insight and demonstrate the feasibility of dual $\mathrm{O}+\mathrm{C}$-band transmission over SSMF. Furthermore, the potential routes to further increase the data rate and the reach of the dual $\mathrm{O}+\mathrm{C}$-band transmission will be discussed after the experimental results presented in Section III.

\section{EXPERIMENTAL RESULTS AND DISCUSSION}

\section{A. Experimental Results}

We first investigated the transmission performance of the four WDM channels in the C-band. Fig. 7 shows the BER versus ROP of the $40-\mathrm{Gb} / \mathrm{s}$ SSB-4QAM-SCM signals. It can be seen that the four channels exhibit comparable BER performance, as expected. For all C-band channels, to ensure a BER lower than the $7 \%$ forward error correction (FEC) limit, i.e., $3.8 \times 10^{-3}$, a ROP of around $-5.5 \mathrm{dBm}$ was required. For reference, the recovered 4QAM constellations at $1549.63 \mathrm{~nm}$ and $1550.23 \mathrm{~nm}$ for an $\mathrm{ROP}=-4.5 \mathrm{dBm}$ are also shown in Fig. 7 and their corresponding BERs were $1.24 \times 10^{-3}$ and $1.56 \times 10^{-3}$, respectively. The corresponding SNR performance of the four channels is presented in Fig. 8. The highest SNR achieved at a ROP of $-1.5 \mathrm{dBm}$ was around $12.5 \mathrm{~dB}$, which is mainly restricted by: (1) the small signal modulation constraint of the DD-MZM to realize the optical SSB signal generation, and (2) the worse sideband suppression ratio performance of the DD-MZM when higher bandwidth is explored $(\sim 22 \mathrm{GHz}$ for the $40-\mathrm{Gb} / \mathrm{s}$ SSB-4QAM-SCM signal), as shown in Fig. 3. Nevertheless, the adoption of the SSB format is beneficial to 
long-distance transmission in the C-band since it offers superior tolerance to the inherent CD effect in the SSMF. Otherwise, there would be several CD-induced spectral nulls within the signal's bandwidth, which lie in and around $8.6 \mathrm{GHz}$, $14.8 \mathrm{GHz}$ and $19.2 \mathrm{GHz}$ over the 50-km length of SSMF (assuming a dispersion value of $17 \mathrm{ps} /(\mathrm{nm} \cdot \mathrm{km})$ and a wavelength of $1550 \mathrm{~nm})$.

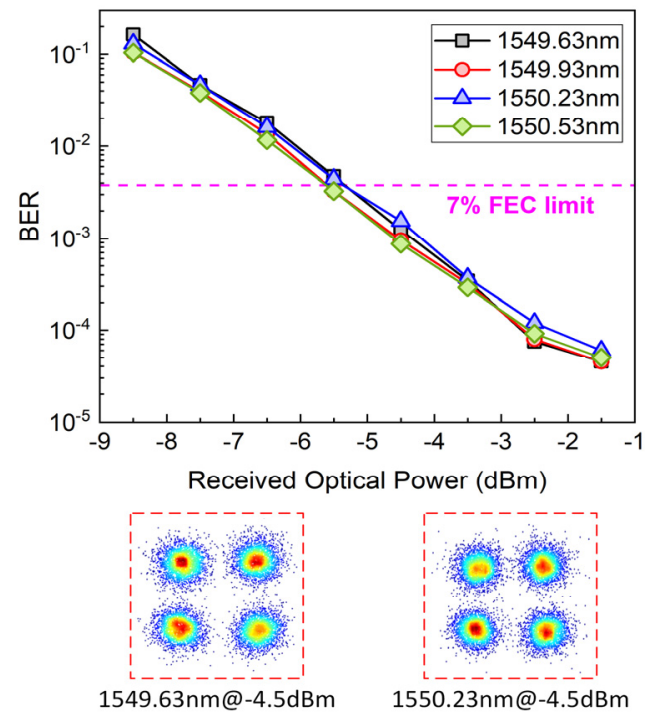

Fig. 7. BER versus ROP of the C-band channels over a 50-km length of SSMF. Insets: recovered 4QAM constellations of the 1549.63-nm and 1550.23-nm channels at $-4.5 \mathrm{dBm}$.

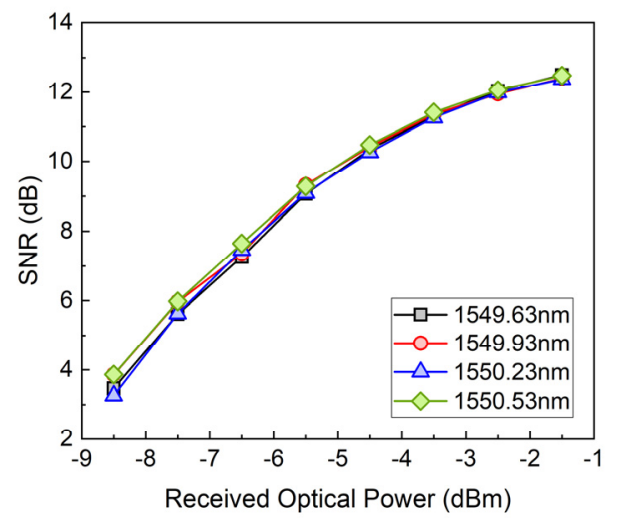

Fig. 8. SNR versus ROP of the C-band channels over a 50-km length of SSMF.

Unlike the C-band case, since DSB signals were adopted in the O-band, their transmission performance will be affected by CD. Although the O-band is widely considered as a low dispersion window, the $\mathrm{CD}$ will still severely limit the usable bandwidth of the overall system, and thus degrade the transmission performance when wideband signals are transmitted over long distances. To characterise the $\mathrm{CD}$-induced power fading in the O-band, we performed simulations for four O-band wavelengths, i.e., $1331 \mathrm{~nm}, 1341$ $\mathrm{nm}, 1351 \mathrm{~nm}$ and $1356 \mathrm{~nm}$, over 50-km and 80-km lengths of SSMF, respectively. The dispersion values at $1331 \mathrm{~nm}, 1341$ $\mathrm{nm}, 1351 \mathrm{~nm}$ and $1356 \mathrm{~nm}$ are assumed to be 2.6, 3, 3.3 and 3.6 $\mathrm{ps} /(\mathrm{nm} \cdot \mathrm{km})$, respectively. Fig. 9 shows the corresponding results. It is clear that with an increase in wavelength, power fading becomes more severe in both cases. For the $50-\mathrm{km}$ case, there is no spectral null for frequencies lower than $20 \mathrm{GHz}$, yet the fading can be up to $\sim 15 \mathrm{~dB}$ at $1356 \mathrm{~nm}$. Further extending the reach to $80 \mathrm{~km}$ will result in deep fading within a $20 \mathrm{GHz}$ bandwidth, as shown in Fig. 9(b). By comparing both cases, it is seen that the fading of the 1331-nm channel is less severe, since it is closer to the zero-dispersion wavelength $(\sim 1310 \mathrm{~nm})$. Therefore, better transmission performance can be expected if shorter wavelengths are available for O-band WDM transmission.
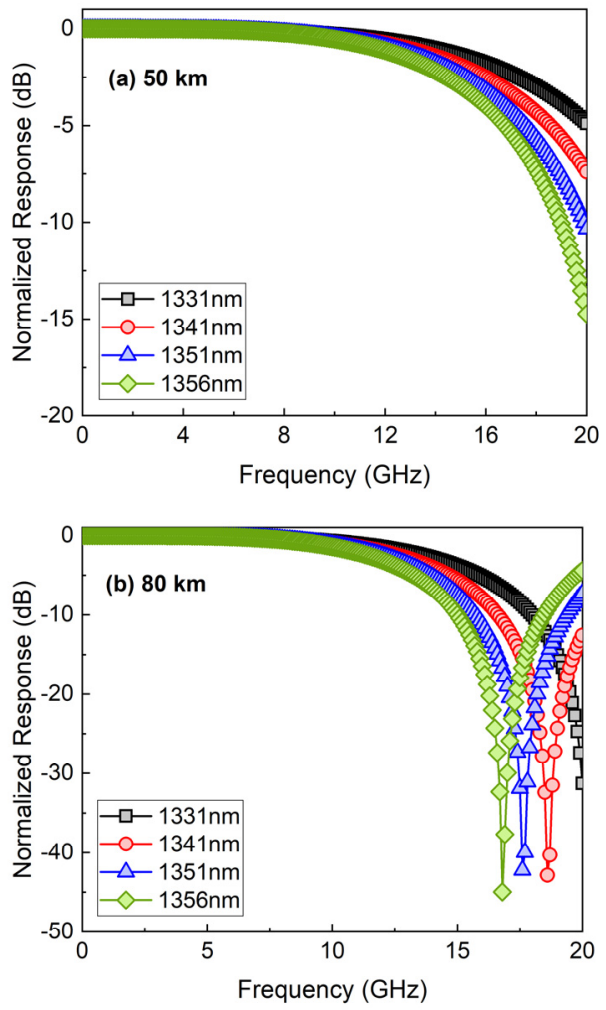

Fig. 9. Simulation results of the CD-induced power fading for O-band wavelengths over SSMF with a length of: (a) $50 \mathrm{~km}$ and (b) $80 \mathrm{~km}$.

Fig. 10 shows the BER performance of the four $40-\mathrm{Gb} / \mathrm{s}$ channels in the O-band (1330.92 nm, $1341.64 \mathrm{~nm}, 1351.32 \mathrm{~nm}$ and $1356.12 \mathrm{~nm}$ ) after a $50-\mathrm{km}$ length of SSMF transmission. It can be seen that the BER performance at longer wavelengths is worse than that at shorter wavelengths, which is attributed to the more severe power fading at these wavelengths, as indicated in Fig. 9. For reference, the recovered OOK eye diagrams for the signals at $1330.92 \mathrm{~nm}$ and $1351.32 \mathrm{~nm}$ at a ROP of $-6 \mathrm{dBm}$ are also given in Fig. 10, and their BERs are $5.08 \times 10^{-4}$ and $4.75 \times 10^{-3}$, respectively. The required ROP to achieve a BER below the 7\% FEC limit is increased from around $-7.5 \mathrm{dBm}$ to around $-5 \mathrm{dBm}$ when the wavelength is increased from $1330.92 \mathrm{~nm}$ to $1356.12 \mathrm{~nm}$. The corresponding SNR performance of the four O-band channels is shown in Fig. 11. Similar to the BER performance, longer wavelengths exhibit worse SNR performance than that of the shorter ones. Moreover, further increasing the ROP leads only to a minor 
improvement in the SNR, since the nonlinearity of the SOA and the CD-induced power fading are the dominant factors that affect the transmission performance rather than the ROP.

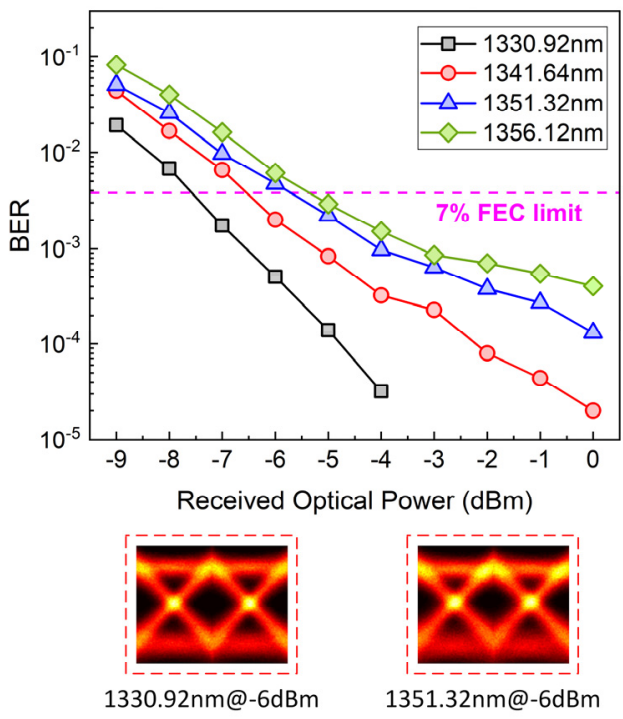

Fig. 10. BER versus ROP of the O-band channels over a $50-\mathrm{km}$ length of SSMF. Insets: recovered OOK eye diagrams of the 1330.92-nm and 1351.32-nm channels at $-6 \mathrm{dBm}$.

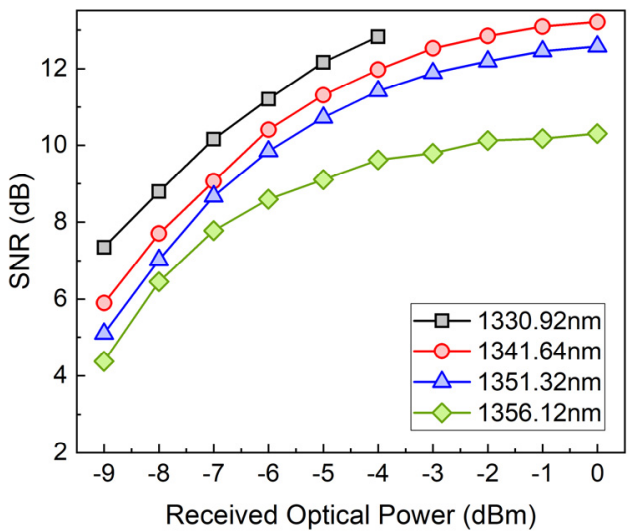

Fig. 11. SNR versus ROP of the O-band channels over a $50-\mathrm{km}$ length of SSMF.

To validate that the performance degradation of longer wavelength channels in the O-band is induced by the $\mathrm{CD}$, a comparison between the RF spectra of the signals in the 1330.92-nm and 1351.32-nm channels is given in Fig. 12. It is clear that for the 1351.32-nm channel, the power fading at high frequencies is more severe after transmission in the $50-\mathrm{km}$ length of SSMF. Furthermore, we have also included the profiles of the simulated frequency responses of the 1331-nm and 1351-nm channels under the effect of CD. As shown in Fig. 12 , the slopes of the simulated profiles agree well with the experimentally detected signals in the two channels, which confirms that the performance degradation of the longer wavelength channels in the O-band is caused by the CD.

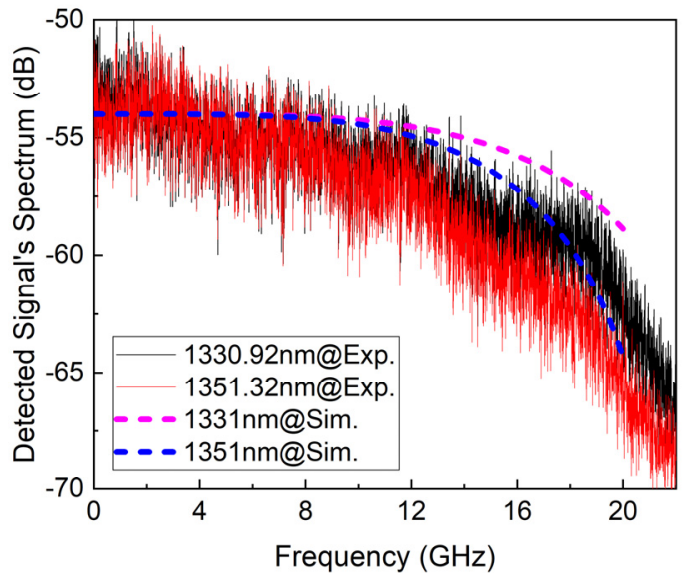

Fig. 12. Comparison of the detected signal's electrical spectrum.

\section{B. Discussion}

By comparing the transmission performance of the two bands, it is seen that the required ROP to achieve a BER below the 7\% FEC limit for the C-band is higher than that of the O-band, even though the responsivity of the PD in the C-band $(0.65 \mathrm{~A} / \mathrm{W})$ is higher than that in the O-band $(0.45 \mathrm{~A} / \mathrm{W})$. This indicates a worse receiver sensitivity in the $\mathrm{C}$-band than in the $\mathrm{O}$-band. The degraded receiver sensitivity is mainly attributed to the relatively high CSPR requirement to fulfil the minimum-phase condition of the KK detection which, in turn, will result in a higher required ROP. It is worth noting that the high CSPR requirement issue can potentially be resolved by using the split-carrier transmitter architecture, as has been demonstrated in [23]. Furthermore, by using the IQM for optical SSB signal generation, the small-signal modulation restriction at the transmitter can also be removed. Both solutions can be beneficial to improve the receiver sensitivity of the C-band transmission. Nevertheless, by sacrificing sensitivity at the receiver, WDM transmission over much longer distances is viable, since the SSB format provides a superior tolerance to $\mathrm{CD}$ and allows a much longer transmission distance by pre-/post-compensating the $\mathrm{CD}$ at the transmitter/receiver. It is worth noting that in this work, the transmission performance in the C-band is mainly restricted by the sideband suppression ratio performance of the used DD-MZM as mentioned above. Both the reach and capacity in the C-band can be further improved by generating the optical SSB-SCM signal with a better DD-MZM or an IQM, as demonstrated in [8].

In contrast, the capacity and reach of the O-band signals in this demonstration were mainly limited by (1) the use of relatively long O-band wavelengths, which were somewhat far away from the zero-dispersion wavelength $(\sim 1310 \mathrm{~nm})$ and (2) the nonlinearity introduced by the SOA, which prevents the use of a higher order modulation format like PAM4 and also limits the achievable reach. In order to further extend the WDM transmission reach in the O-band, either the adopted channels need to be closer to the zero-dispersion wavelength or SSB formats should also be utilised if sufficient power budget is available. Since enhanced amplification capability and superior nonlinear performance are offered by the BDFA compared to 
the SOA [20, 24-25], longer reach with higher capacity should be also realistically achievable in the O-band by replacing the booster SOA with a BDFA, which will be further investigated in due course.

\section{CONCLUSION}

In this paper, 320-Gb/s dual O+C-band DD transmission with eight WDM channels over a 50-km length of SSMF was experimentally demonstrated. $4 \times 40-\mathrm{Gb} / \mathrm{s}$ SSB-4QAM-SCM with $\mathrm{KK}$ detection and $4 \times 40-\mathrm{Gb} / \mathrm{s}$ Nyquist-OOK were used in the C- and O-band, respectively. The results showed that the O-band offered better receiver sensitivity than that of the $\mathrm{C}$-band, despite the corresponding responsivity of the PD being lower. However, the transmission performance in the O-band was restricted by the CD impairment, especially when longer O-band wavelengths and longer reach were explored. In contrast, the C-band showed better tolerance to CD effects thanks to the adoption of the SSB format. However, the C-band transmission showed inferior receiver sensitivity compared with the O-band transmission, which resulted from the high CSPR requirement of the KK detection and the small-signal modulation restriction when using the DD-MZM to generate optical SSB signals. The demonstrations in this work showed the possibility to extend the reach of currently deployed O-band systems for intermediate- to long-haul applications, wherein both $\mathrm{O}$ - and $\mathrm{C}$-band can be used for high-capacity WDM transmission.

\section{ACKNOWLEDGMENT}

The data for this work is accessible through the University of Southampton Institutional Research Repository (DOI: 10.5258/SOTON/D0936).

\section{REFERENCES}

[1] E. Agrell, M. Karlsson, A.R. Chraplyvy, et al., "Roadmap of optical communications," J. Opt., vol. 18, pp. 1-40, 2016.

[2] K. Zhong, X. Zhou, J. Huo, et al., "Digital Signal Processing for Short-Reach Optical Communications: A Review of Current Technologies and Future Trends," J. Light. Technol., vol. 36, no. 2, pp. 377-400, 2018.

[3] K. Kikuchi, "Fundamentals of Coherent Optical Fiber Communications," J. Light. Technol., vol. 34, no. 1, pp. 157-179, 2016.

[4] W. Peng, X. Wu, V.R. Arbab, et al., "Theoretical and Experimental Investigations of Direct-Detected RF-Tone-Assisted Optical OFDM Systems," J. Light. Technol., vol. 27, no. 10, pp. 1332-1339, 2009.

[5] S. Randel, D. Pilori, S. Chandrasekhar, et al., " $100-\mathrm{Gb} / \mathrm{s}$ discrete-multitone transmission over $80-\mathrm{km} \mathrm{SSMF}$ using single-sideband modulation with novel interference-cancellation scheme," Proc. of ECOC, Valencia, Spain, paper Mo.4.5.2, 2015.

[6] X. Chen, C. Antonelli, S. Chandrasekhar, et al., "Kramers-Kronig Receivers for 100-km Datacenter Interconnects," J. Light. Technol., vol. 36 , no. 1 , pp. 79-89, 2018.
[7] Z. Li, M.S. Erkılınç, S. Pachnicke, et al., "Signal-signal Beat Interference Cancellation in Spectrally-efficient WDM Direct-detection Nyquist-pulse-shaped 16-QAM Subcarrier Modulation," Opt. Express, vol. 23, no. 18, pp. pp. 23694-23709, 2015.

[8] Z. Li, M.S. Erkılınç, K. Shi, et al., "SSBI Mitigation and the Kramers-Kronig Scheme in Single-Sideband Direct-Detection Transmission with Receiver-Based Electronic Dispersion Compensation,” J. Light. Technol., vol. 35, no. 10, pp. 1887-1893, 2017.

[9] A. Mecozzi, C. Antonelli, M. Shtaif, "Kramers-Kronig coherent receiver," Optica, vol. 3, no. 11, pp. 1220-1227, 2016.

[10] T. Bo, H. Kim, "Toward Practical Kramers-Kronig Receiver: Resampling, Performance, and Implementation," J. Light. Technol., vol. 37, no. 2, pp. 461-469, 2019.

[11] T. Bo, H. Kim, "Kramers-Kronig Receiver Operable Without Digital Upsampling," Opt. Express, vol. 26, no. 11, pp. 13810-13818, 2018.

[12] C. Sun, D. Che, H. Ji, et al., "Towards Low Carrier-to-Signal Power Ratio for Kramers-Kronig Receiver," Proc. of OFC, San Diego, USA, paper M1H.6, 2019.

[13] J.K. Fischer, M. Cantono, V. Curri, et al., "Maximizing the Capacity of Installed Optical Fiber Infrastructure via Wideband Transmission," Proc. of ICTON, Bucharest, Romania, paper Tu.B3.3, 2018.

[14] F. Hamaoka, M. Nakamura, S. Okamoto, et al., "Ultra-Wideband WDM Transmission in S-, C-, and L-Bands Using Signal Power Optimization Scheme," J. Light. Technol., vol. 37, no. 8, pp. 1764-1771, 2019.

[15] S. Okamoto, K. Horikoshi, F. Hamaoka, et al., "5-band (O, E, S, C, and L) WDM Transmission with Wavelength Adaptive Modulation Format Allocation," Proc. ECOC, Dusseldorf, Germany, pp. 1172-1174, 2016.

[16] Chris Cole, "1310 nm vs $1550 \mathrm{~nm}$," presented at ECOC, Gothenburg, Sweden, 2017. [Online]. Available: https://www.finisar.com/sites/ default/files/resources/1310nm_vs_1550nm_ecoc2017.pdf.

[17] N. Taengnoi, K.R.H. Bottrill, N.K. Thipparapu, et al., "Amplified O-Band WDM Transmission Using a Bi-Doped Fibre Amplifier," Proc. of ECOC, Rome, Italy, paper Mo3E.2, 2018.

[18] E.M. Dianov., "Amplification in Extended Transmission Bands Using Bismuth-Doped Optical Fibers," J. Light. Technol., vol. 31, no. 4, pp. 681-688, 2013.

[19] N. K. Thipparapu, A. A. Umnikov, P. Barua, et al., "Bi-doped fiber amplifier with a flat gain of $25 \mathrm{~dB}$ operating in the wavelength band 1320-1360 nm," Opt. Lett., vol. 41, no. 7, pp. 1518-1521, 2016.

[20] N. Taengnoi, K.R.H. Bottrill, N.K. Thipparapu, et al., "WDM Transmission With In-Line Amplification at $1.3 \mu \mathrm{m}$ Using a Bi-Doped Fiber Amplifier," J. Light. Technol., vol. 37, no. 8, pp. 1826-1830, 2019.

[21] L. Zhang, T. Zuo, Y. Mao, et al., "Beyond 100-Gb/s Transmission Over 80-km SMF Using Direct-Detection SSB-DMT at C-Band," J. Light. Technol., vol. 34, no. 2, pp. 723-729, 2016.

[22] N. Taengnoi, K. R. H. Bottrill, Y. Hong, et al., "Reach extension of PAM4 signals in O-band transmission by application of alternate-mark-inversion," in Proc. of ECOC, Dublin, Ireland, P93, 2019

[23] T. Gerard, Z. Liu, M. S. Erkılınç, et al., "Intra-Datacentre Links Using a Split-Carrier Transmitter Architecture," IEEE Photon. J., vol. 11, no. 4, pp. 7204115, 2019.

[24] M. A. Melkumov, V. Mikhailov, A. M. Khegai, et al., "25 Gbs ${ }^{-1}$ Data Transmission using A Bismuth-doped Fibre Amplifier with A Gain Peak Shifted to 1300 nm," Quantum Electron., vol. 48, no. 11, pp. 989-992, 2018.

[25] V. Mikhailov, M. A. Melkumov, D. Inniss, et al., "Simple Broadband Bismuth Doped Fiber Amplifier (BDFA) to Extend O-band Transmission Reach and Capacity," Proc. of OFC, San Diego, USA, paper M1J.4, 2019. 